OPEN ACCESS

Edited by:

Ryan J. Stark,

Vanderbilt University Medical Center,

United States

Reviewed by:

Luca Spiezia

University of Padua, Italy

Gail Mary Annich,

Hospital for Sick Children, Canada

${ }^{*}$ Correspondence:

Nguyen Thi Thu Ha

nthha.hmu@gmail.com

tThese authors have contributed equally to this work and share first authorship

Specialty section This article was submitted to Pediatric Critical Care, a section of the journal Frontiers in Pediatrics

Received: 05 March 2021 Accepted: 10 May 2021

Published: 02 June 2021

Citation:

Tuan TA, Ha NTT, Xoay TD, My TTK

Nghiem LT and Dien TM (2021)

Hypocoagulable Tendency on Thromboelastometry Associated With Severity and Anticoagulation Timing in Pediatric Septic Shock: A Prospective Observational Study.

Front. Pediatr. 9:676565. doi: 10.3389/fped.2021.676565

\section{Hypocoagulable Tendency on Thromboelastometry Associated With Severity and Anticoagulation Timing in Pediatric Septic Shock: A Prospective Observational Study}

\author{
Ta Anh Tuan ${ }^{1,2 t}$, Nguyen Thi Thu Ha ${ }^{1 * t}$, Tran Dang Xoay ${ }^{1}$, Tran Thi Kieu My ${ }^{3}$, \\ Luong Thi Nghiem ${ }^{4}$ and Tran Minh Dien ${ }^{2,5}$ \\ ${ }^{1}$ Pediatric Intensive Care Unit, Vietnam National Children's Hospital, Hanoi, Vietnam, ${ }^{2}$ University of Medicine and Pharmacy, \\ Vietnam National University, Hanoi, Vietnam, ${ }^{3}$ Division of Hematology, Hanoi Medical University, Hanoi, Vietnam, \\ ${ }^{4}$ Hematology Department, Vietnam National Children's Hospital, Hanoi, Vietnam, ${ }^{5}$ Surgical Intensive Care Unit, Vietnam \\ National Children's Hospital, Hanoi, Vietnam
}

Objective: To identify whether coagulation profiles using thromboelastometry are associated with outcomes in pediatric septic shock. The primary outcomes were the development of disseminated intravascular coagulation (DIC) and the severity of the pediatric intensive care unit (PICU) existing scoring systems, while the secondary outcome was hospital mortality. This study aimed to contribute to current findings of the limitations of conventional tests in determining the optimal timing of anticoagulation in sepsis.

Design: A prospective, observational study conducted between August 2019 and August 2020.

Setting: PICU at a pediatric tertiary hospital in Hanoi, Vietnam.

Patients: Fifty-five pediatric patients who met the septic shock criteria were enrolled.

Measurements and Main Results: Fifty-five patients with septic shock were recruited. At the time of diagnosis, thromboelastometry revealed normocoagulability, hypercoagulability, and hypocoagulability in 29,29 , and $42 \%$ of the patients, respectively ( $p$ > 0.05); however, most patients in the overt DIC and non-survival groups progressed to hypocoagulability (82 and 64\%, respectively). The overt DIC, PELOD-2 > 8, PRISM-III $>11$, and non-survival group had a significant hypocoagulable tendency according to thromboelastometry parameters [prolonged clotting time (CT) and clot formation time (CFT); and reduced $\alpha$-angle ( $\alpha$ ), maximum clot firmness (MCF), thrombodynamic potential index (TPI)] compared to the non-overt DIC, PELOD-2 $\leq 8$, PRISM-III score $\leq 11$ and survival group $(p<0.05)$. Conventional parameters between the normocoagulable and hypercoagulable groups were not different $(p>0.05)$. Hypocoagulability was characterized by lower platelet count and fibrinogen level, higher prolonged prothrombin time (PT), international normalized ratio (INR), and activated partial thromboplastin time (APTT), and higher D-dimer level than in hypercoagulability $(p<0.05)$. 
Hypocoagulable tendency on thromboelastometry had a higher hazard at a PT $>16.1 \mathrm{~s}$ [area under the curve $(\mathrm{AUC})=0.747$, odds ratio $(\mathrm{OR})=10.5, p=0.002$ ], INR $>1.4$ $(\mathrm{AUC}=0.754, \mathrm{OR}=6.9, p=0.001)$, fibrinogen $<3.3 \mathrm{~g} / \mathrm{L}(\mathrm{AUC}=0.728, \mathrm{OR}=9.9$, $p=0.004$ ), and D-dimer $>3,863 \mathrm{ng} / \mathrm{mL}(\mathrm{AUC}=0.728, \mathrm{OR}=6.7, p=0.004$ ).

Conclusions: Hypocoagulable tendency using thromboelastometry is associated with the severity of septic shock. Conventional coagulation tests may fail to detect hypercoagulability, which is crucial in determining anticoagulation timing.

Keywords: hypocoagulable, pediatric, septic shock, thromboelastometry, PELOD-2, PRISM-III

\section{INTRODUCTION}

Sepsis-induced coagulopathy (SIC) is initially triggered by the combination of procoagulant upregulation, endogenous anticoagulant downregulation, and fibrinolytic impairment. These mechanisms are beneficial host responses that contribute to a hypercoagulable state, which is termed "immunothrombosis"-a defense against the spread of pathogens into the nearby tissues or systemic circulation at an early stage of sepsis (1-3). However, its dysregulation leads to overwhelming activation of coagulation that induces microthrombosis, particularly disseminated intravascular coagulation (DIC) and multiple organ dysfunction syndrome (MODS). The ongoing excessive consumption of coagulation factors ultimately results in a hypocoagulable state with a high risk of severe hemorrhagic complications $(2,4,5)$. Previous studies have reported that the early phase of sepsis is characterized by hypercoagulability, whereas the later stages (severe sepsis or septic shock) trend toward hypocoagulability and are associated with increased morbidity and mortality $(1,6-12)$. Therefore, proper identification of the coagulation profile is pivotal for appropriate treatment and prognosis. The timing of coagulation-targeting therapy at the hypercoagulable stage may reduce the risk of disseminative thrombosis and progression of MODS. In contrast, blood transfusion therapy is indicated during hypocoagulability with a high risk of bleeding because of the depleted components of the coagulation system $(2,13)$. However, the current standards for hemostatic assessment are generally based on conventional parameters with several limitations. As they are performed using plasma, the function of cell components (such as the contribution of platelets to thrombosis) in the coagulation system is bypassed. Additionally, these parameters are not reflective of the in vivo hemostatic process and do not provide qualitative or functional data $(1,14)$. Moreover, their inability to detect hypercoagulable status and fibrinolytic activity has been revealed $(15,16)$. Hence, methodological improvements in thromboelastography (TEG) or rotational thromboelastometry (ROTEM) have been widely utilized as point-of-care tests for optimal hemostatic resuscitation in patients with sepsis. These tests measure dynamic global clotting and overcome limitations of the conventional assay in discriminating between hyper- and hypocoagulability $(10-12,17)$. Hypocoagulable profile in viscoelastic parameters is associated with poor outcomes (11); therefore, clinicians should monitor the patients more strictly and adopt adequate diagnostic and therapeutic strategies earlier. Furthermore, the efficacy of the viscoelastic test for the guidance of blood transfusion practice has been reported $(15,16)$. Prior studies using global clotting assay proved its ability to evaluate coagulation and prognosis. However, these data were mainly obtained in adults and cases of non-septic shock, and the broader use of ROTEM remained controversial $(8,15)$; therefore, additional studies are required. Our study aimed to evaluate hemostatic disturbance using the ROTEM assay and identify the association between these parameters and the severity of septic shock in pediatric patients. Additionally, we aimed to contribute to the current findings on the limitations of conventional coagulation tests in optimal anticoagulation timing in sepsis.

\section{MATERIALS AND METHODS}

\section{Ethics Approval}

Ethics approval for our study was obtained from the Institutional Review Board of Vietnam National Children's Hospital (approval no. 197-BVNTW-VNCSKTE).

\section{Patients}

This was a single-center prospective observational study from August 2019 to August 2020 at the pediatric intensive care unit (PICU) of a tertiary hospital in Vietnam. Patients aged 1 month-18 years with septic shock, based on the criteria of the International Sepsis Definitions Conference 2005, were recruited (18). Patients with a history of coagulopathy, those undergoing anticoagulant treatment, or those who died within $24 \mathrm{~h}$ of admission were excluded from this study.

Patient demographic, clinical, and laboratory variables were collected. Demographic variables included age, sex, weight, and underlying conditions. Clinical variables included temperature, heart rate, ventilatory condition, mean arterial pressure, consciousness level evaluated according to the Glasgow Coma Scale (19), and hemorrhagic or thrombotic events. Laboratory variables included white blood count $\left(\times 10^{9} / \mathrm{L}\right)$, hemoglobin $(\mathrm{g} / \mathrm{L})$, C-reactive protein $(\mathrm{mg} / \mathrm{L})$, lactate level $(\mathrm{mmol} / \mathrm{L})$, urea $(\mathrm{mmol} / \mathrm{L})$, creatinine $(\mu \mathrm{mol} / \mathrm{L})$, aspartate aminotransferase $(\mathrm{IU} / \mathrm{L})$, alanine aminotransferase (IU/L), albumin $(\mathrm{g} / \mathrm{L})$, protein $(\mathrm{g} / \mathrm{L})$, direct and indirect bilirubin $(\mathrm{mg} / \mathrm{dL})$, and blood culture results. Pediatric Risk of Mortality III (PRISM-III) and Pediatric Logistic Organ Dysfunction-2 (PELOD-2) scores at 24-hour after septic shock diagnosis were calculated $(20,21)$. 


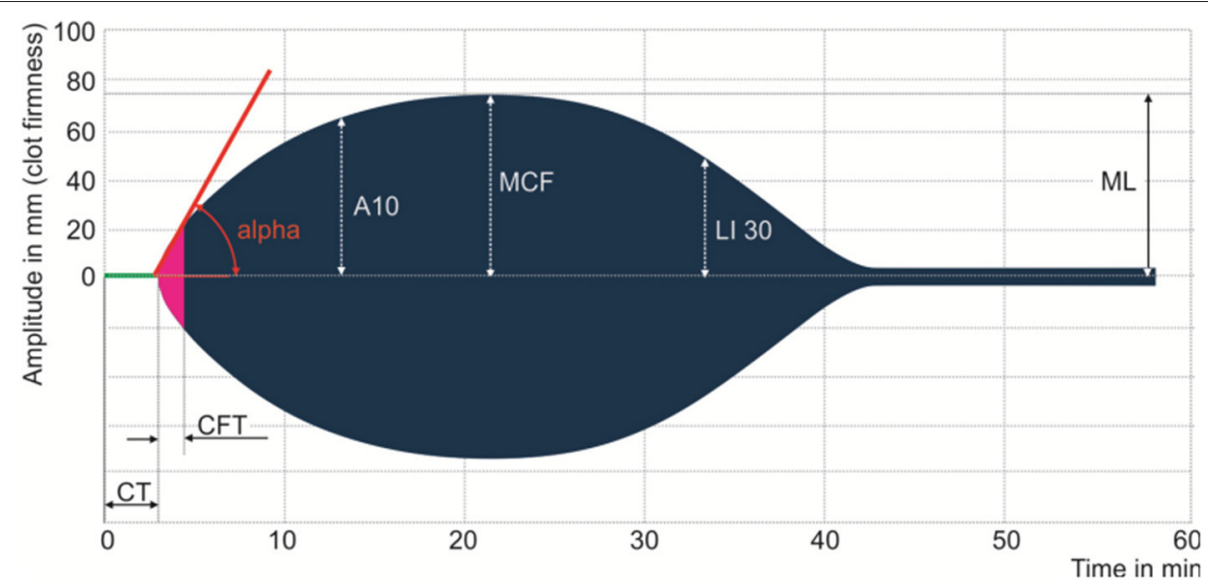

FIGURE 1 | Main parameters of the rotational thromboelastometry test. Clotting time (CT), clot formation time (CFT), alpha angle (ALP), the amplitude after 10 min after CT, maximum clot firmness (MCF), clot lysis index at 30 min after CT (LI30), and maximum lysis (ML).

\section{Conventional Coagulation Parameters and DIC Score}

The conventional coagulation parameters at the time of diagnosis of septic shock were platelet count (PLT) $\left(\times 10^{9} / \mathrm{L}\right)$ [using K4500 hematology analyzer (Sysmex, Kobe, Japan)], prothrombin time (PT) (s), international normalized ratio (INR), activated partial thromboplastin time (APTT) (s), fibrinogen $(\mathrm{g} / \mathrm{L})$, and D-dimer (ng/mL FEU) [using ACL TOP 750-CTS coagulation analyzer (Instrument Laboratory, American)]. DIC score was calculated according to the International Society of Thrombosis and Hemostasis (ISTH) criteria, 2001: (1) PLT count from 50 to $100 \times 10^{9} / \mathrm{L}(1$ point $),<50 \times 10^{9} / \mathrm{L}$ ( 2 points); (2) INR from 1.4 to 2.3 ( 1 point), $>2.3$ ( 2 points); (3) fibrinogen $<1 \mathrm{~g} / \mathrm{L}$ (1 point); and (4) D-dimer from 399 to $4,000 \mathrm{ng} / \mathrm{mL}$ (2 points), $>4,000 \mathrm{ng} / \mathrm{mL}$ (3 points). Patients had overt-DIC if the DIC score was $\geq 5$ (4).

\section{Rotation Thromboelastometry}

Samples of whole blood anticoagulated with $3.8 \%$ sodium citrate were collected at the time of diagnosis and analyzed with ROTEM delta analyzer $\left(\right.$ ROTEM $^{\circledR}$, TEM International $\mathrm{GmbH}$, Munich, Germany). Four main tests were performed: EXTEM (the extrinsic pathway); INTEM (the intrinsic pathway); FIBTEM (the contribution of fibrinogen to the clot); and APTEM (assessment of hyperfibrinolysis) (22, 23). Each test records the kinetic changes in a sample of whole blood during clot formation and when the clot retracts and/or lyses. As external pathway activation plays a crucial role in SIC, our study focused on EXTEM parameters, including clotting time (CT)-time to thrombin generation, clot formation time (CFT), and $\alpha$ angle $(\alpha)$-fibrin polymerization and clot formation, maximum clot firmness (MCF) - the measure of maximum clot amplitude describing clot stability and thrombodynamic potential index (TPI) calculated by $30^{*}\left(100^{*} \mathrm{MCF}\right) / \mathrm{CFT} *(100-\mathrm{MCF})$ describing global coagulation. Three coagulation profiles were categorized on EXTEM, including normocoagulability, hypercoagulability, and hypocoagulability. Hypercoagulability was defined as at least one of shortened CT or CFT, elevated $\alpha$, MCF, or TPI. Hypocoagulability was defined as at least one of prolonged CT or CFT, reduced $\alpha, \mathrm{MCF}$, or TPI. The reference range of the aforementioned EXTEM parameters were determined by a multicenter investigation of healthy volunteers $(24,25)$. Figure 1 is a diagram illustrating the main parameters of the rotational thromboelastomatry test.

\section{Statistical Analysis}

Statistical analyses were performed using SPSS software version 20.0 software (IBM Corporation, Armonk, NY). Categorical variables are described as frequency and percentage, whereas continuous variables are expressed as medians and interquartile ranges (IQRs). We used the Mann-Whitney $U$ test to compare the mean of non-parametric variables between the two groups; Chi-square test was used to test the equality of independent proportions. The receiver operating characteristic (ROC) curve with the Younden index was used to identify the performance and cutoff point of conventional parameters on discrimination in coagulation profiles. Area under the ROC (AUC) curve values that fell between 0.7 and 0.8 were acceptable, between 0.8 and 0.9 were excellent, and $>0.9$ were outstanding (26). A two-sided $p$-value of $<0.05$ was considered statistically significant.

\section{RESULTS}

\section{Patients}

Fifty-five patients (31 boys and 24 girls) were recruited in this study. The median age was 7 (range: 1-205) months, and $13 \%$ had comorbidities. The frequency of clinical bleeding and thrombosis was 20 and 2\%, respectively. Of the total, 31\% presented with positive blood cultures, and the most prevalent organisms isolated were Streptococcus pneumonia, Staphylococcus aureus, and Escherichia coli. The median PELOD-2 score and PRISM-III scores were 8 and 11, respectively. At the time of diagnosis, the prevalence of overt-DIC was $20 \%$. The mortality 
TABLE 1 | Patient characteristics.

\begin{tabular}{|c|c|}
\hline Characteristics & Value $(n=55$ \\
\hline \multicolumn{2}{|l|}{ Demographic data } \\
\hline Age, months (Median, range) & $7(1-205)$ \\
\hline Male sex $(n, \%)$ & $31(56 \%)$ \\
\hline Underlying conditions ( $n, \%)$ & $7(13 \%)$ \\
\hline \multicolumn{2}{|l|}{ Clinical presentations of hemostatic abnormalities $(n, \%)$} \\
\hline Bleeding & $11(20 \%)$ \\
\hline Thrombosis & $1(2 \%)$ \\
\hline Positive blood culture ( $n, \%)$ & $17(31 \%)$ \\
\hline Streptococcus pneumoniae & $4(24 \%)$ \\
\hline Staphylococcus aureus & $4(24 \%)$ \\
\hline Escherichia coli & $4(24 \%)$ \\
\hline Enterobacter cloacae & $1(6 \%)$ \\
\hline Burkholderia pseudomallei & $1(6 \%)$ \\
\hline Burkholderia cepacia & $1(6 \%)$ \\
\hline Pseudomonas aeruginosa & $1(6 \%)$ \\
\hline Klebsiella pneumoniae & $1(6 \%)$ \\
\hline Kodamaea ohmeri & $1(6 \%)$ \\
\hline VIS score $(n, \%)$ & $55(100 \%)$ \\
\hline At 24-h post PICU admission (Median, IQR) & $20(7-43)$ \\
\hline PELOD-2 score (Median, IQR) & $8(6-11)$ \\
\hline PRISM-III score (Median, IQR) & $11(7-18)$ \\
\hline Overt DIC at diagnostic time $(n, \%)$ & $11(20 \%)$ \\
\hline Non-invasive mechanical ventilation ( $n, \%)$ & $7(13 \%)$ \\
\hline Invasive mechanical ventilation (n, \%) & $48(87 \%)$ \\
\hline Length of invasive mechanical ventilation (days), median (IQR) & $5(3-7)$ \\
\hline PICU length of stay (days), median (IQR) & $14(5-20)$ \\
\hline Mortality (n, \%) & $17(31 \%)$ \\
\hline
\end{tabular}

Data are presented as median (IQR: Q1-Q3) or number (\%).

IQR, Interquartile Range; PELOD-2, Pediatric Logistic Organ Dysfunction-2; PRISM-III, Pediatric Risk of Mortality Score III; DIC, Disseminated intravascular coagulation; PICU, pediatric intensive care unit.

rate of the overt-DIC group was 55\%, while the overall mortality rate was $31 \%$ (Table $\mathbf{1}$ ).

\section{Conventional Coagulation Data}

Supplementary Table enlists that the overt-DIC, PELOD-2 > 8 , and PRISM-III > 11 group had lower platelet count and fibrinogen level, more prolonged PT, INR, APTT, and higher D-dimer than their counterparts, the non-overt DIC, PELOD-2 $\leq 8$, and PRISM-III $\leq 11$ group $(p<0.05)$. The difference in these parameters between the survivors and non-survivors was not significant $(p>0.05)$.

\section{Rotational Thromboelastometry Data}

Coagulation profiles on EXTEM are displayed in Figure 2. Overall, the proportion of coagulation profiles was not different ( $p>0.05$ ), with 29,29 , and $42 \%$ being normocoagulable, hypercoagulable, and hypocoagulable, respectively. However, subgroup analyses revealed that most patients with overt-DIC and non-survival progressed to the hypocoagulable state at the time of diagnosis $[p<0.05$ (82 and $64 \%$, respectively)].
The overt-DIC, PELOD- $2>8$, PRISM-III > 11, and nonsurvival group had significant hypocoagulable tendencies in the studied parameters (prolonged CT, CFT and reduced $\alpha, \mathrm{MCF}$, TPI) compared with those of the non-overt DIC, PELOD$2 \leq 8$, PRISM-III $\leq 11$, and survival group $(p<0.05)$ (Supplementary Table).

\section{Conventional Coagulation Tests Failed to Detect the Hypercoagulable}

In this study, we compared the conventional parameters among subgroups of coagulation profiles on EXTEM (normocoagulability, hypercoagulability, and hypocoagulability) (Table 2). An insignificant difference in most parameters (PT, INR, APTT, fibrinogen, D-Dimer) was observed between the normocoagulable and hypercoagulable groups $(p>0.05)$. Hypocoagulability was associated with a lower platelet count and fibrinogen level, but more prolonged PT, INR, APTT, and higher D-dimer level than hypercoagulability $(p<0.05)$.

Further analyses revealed that the hypocoagulable tendency on thromboelastometry had a higher hazard at a PT $>16.1 \mathrm{~s}$ [AUC $=0.747$, odds ratio $(\mathrm{OR})=10.5, p=0.002], \mathrm{INR}>1.4$ $(\mathrm{AUC}=0.754, \mathrm{OR}=6.9, p=0.001)$, fibrinogen $<3.3 \mathrm{~g} / \mathrm{L}(\mathrm{AUC}$ $=0.728, \mathrm{OR}=9.9, p=0.004)$, and D-dimer $>3,863 \mathrm{ng} / \mathrm{mL}$ $(\mathrm{AUC}=0.728, \mathrm{OR}=6.7, p=0.004)($ Table 3$)$.

\section{DISCUSSION}

This study determined the relationship between hemostatic disturbance from viscoelastic assay and severity in 55 pediatric patients with septic shock at the PICU of the Vietnam National Children's Hospital. To the best of our knowledge, this is the first report from a developing country describing the association between hypocoagulation profile based on thromboelastometry and the severity of septic shock in a pediatric population; additionally, this study further strengthened the findings of previous reports on the drawbacks of conventional coagulation tests.

Previous studies in adults reported the detection of hypercoagulability on viscoelastic assay as ranging from 30 to $100 \%$ (17). Hypercoagulability was observed in the early stage of sepsis, consistent with the aforementioned hypothesis of "immunothrombosis" defense (1, 3, 15). The development of hypocoagulability is commonly associated with the increasing severity of each stage (severe sepsis and septic shock) $(12,15,16,27)$. Previous studies observed hemostatic response by a rapid increase in clot mass development (with higher $\alpha$ and MCF), possibly indicating a hypercoagulable phase and potential thromboembolic risk in the early stage of sepsis followed by the progression of hypocoagulable trends (with prolonged CT and CFT) in the later phase $(6,28)$. In a previous study on adult sepsis, TEG profile of the septic shock group at the time of intensive care unit (ICU) entry and $6 \mathrm{~h}$ after ICU entry significantly tended to be more hypocoagulable than that of the sepsis group (12). Similarly, our result showed that most patients were in the hypocoagulable stage on EXTEM at the time 


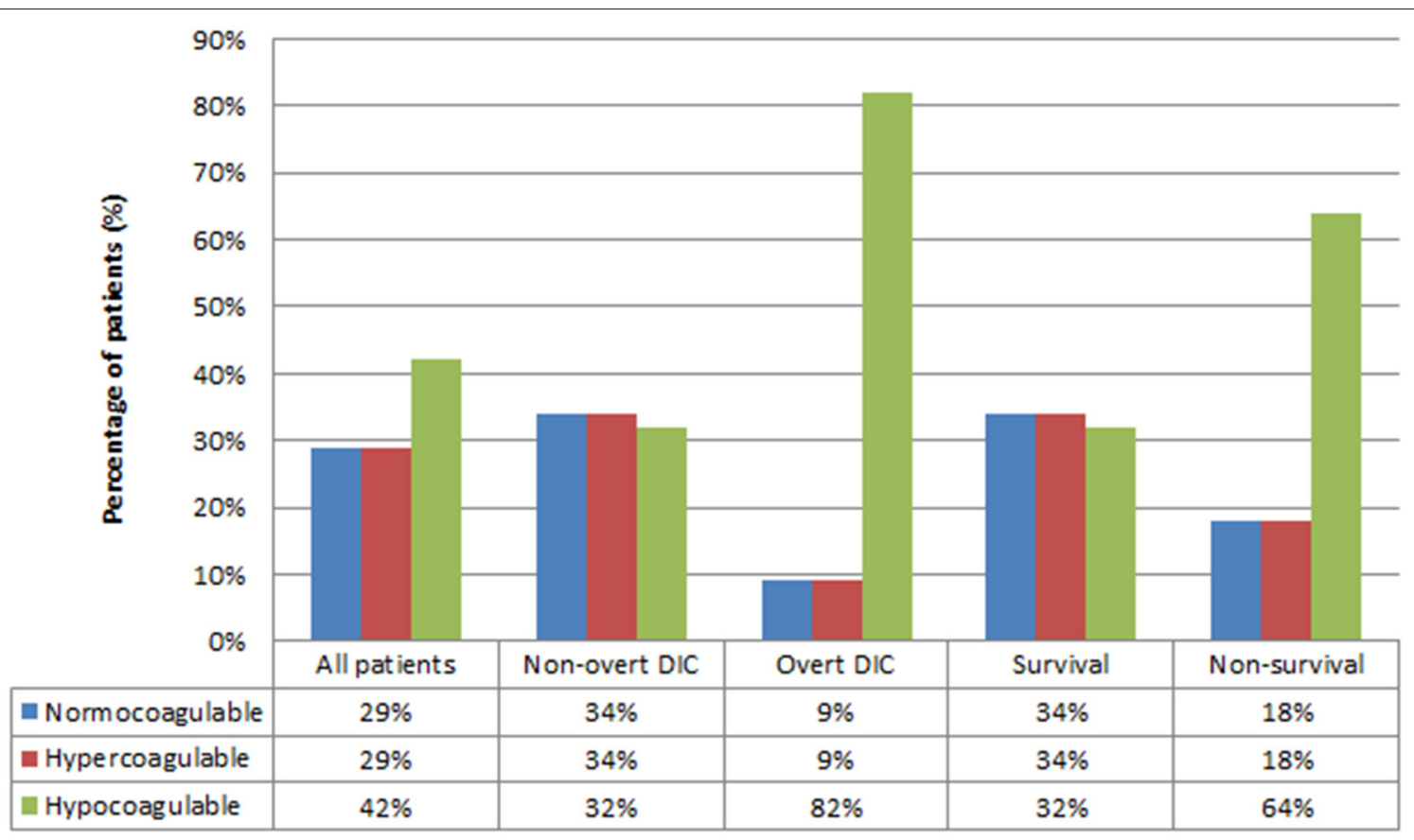

FIGURE 2 | Coagulation profiles based on rotational thromboelastometry test. DIC, Disseminated intravascular coagulation. $P$-value for Chi-square test of three coagulation profiles for all patients $=0.41$, non-overt $\mathrm{DIC}=0.978$, overt-DIC $=0.003$, survival $=0.974$, non-survival $=0.023$.

TABLE 2 | Performance of conventional parameters in the discrimination of coagulation status based on thromboelastometry.

\begin{tabular}{|c|c|c|c|c|c|c|}
\hline Parameters(Median, IQR) & Normocoagulable $(n=16)$ & Hypercoagulable( $(n=16)$ & Hypocoagulable $(n=23)$ & $p^{a}$ & $p^{b}$ & $p^{c}$ \\
\hline $\operatorname{PLT}\left(\times 10^{9} / \mathrm{L}\right)$ & $227(109-365)$ & $474(300-549)$ & $177(66-253)$ & 0.009 & $<0.001$ & 0.242 \\
\hline PT (s) & $15(14-29.6)$ & $13.9(12.7-18.7)$ & 20 (16.2-26.3) & 0.356 & 0.003 & 0.028 \\
\hline INR & $1.3(1.1-1.6)$ & $1.2(1-1.5)$ & $1.6(1.4-2.4)$ & 0.327 & 0.003 & 0.019 \\
\hline APTT (s) & $40.7(37-45.3)$ & 36.9 (31.8-51.3) & $48.3(38-65.6)$ & 0.546 & 0.050 & 0.056 \\
\hline Fibrinogen (g/L) & $4(3.7-5)$ & $3.9(2.9-5.4)$ & $2.6(1.5-4.2)$ & 0.821 & 0.035 & 0.007 \\
\hline D-Dimer (ng/mL) & $2,664(1,954-5,281)$ & $2,096(974-3,381)$ & $6,935(2,343-17,172)$ & 0.200 & 0.006 & 0.044 \\
\hline
\end{tabular}

Data are presented as median (IQR: Q1-Q3).

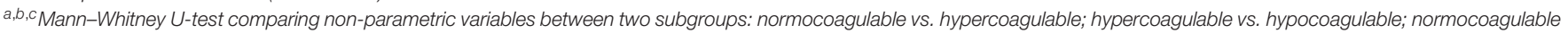
vs. hypocoagulable, respectively. Bold values indicate the statistically significant ( $p$-value $<0.05$ ).

IQR, Interquartile range; PLT, Platelet; PT, Prothrombin Time; INR, International Normalized Ratio; APTT, Activated Partial Thromboplastin Time.

of septic shock diagnosis; however, the difference among profiles was insignificant $(p>0.05)$ (Figure 2).

Interestingly, the highest prevalence of hypocoagulability was observed in the overt-DIC and non-survival groups on subgroup analyses in this study (Figure 2). The non-overt DIC group was mostly hypercoagulable, while progression to hypocoagulability was observed in the overt-DIC group (22). We hypothesized that most cases who met the overt-DIC criteria developed hypocoagulability secondary to the depletion of coagulation factors; therefore, using the ISTH scoring system may delay the early-stage diagnosis of septic coagulopathy. Other studies, mainly in adult populations, also revealed that hypocoagulability was characterized by high CT and CFT and low MCF in patients with overt-DIC compared to patients with non-overt DIC $(22,29,30)$. Depleted coagulation factors during DIC can be a reasonable explanation for the prolonged CT and CFT.
Lower MCF in overt DIC was induced by thrombocytopenia and hypofibrinogenemia, which are two main factors that influence the firmness of clots. Similarly, various studies reported that hypocoagulability was associated with higher consumption of blood products and lower survival. Previous studies identifying parameters with the highest prognostic value of mortality have been reported; however, the results remain unclear $(9-12,17$, $31,32)$. In addition, the data in the pediatric population were limited; hence, further investigations are warranted to clarify this issue. Further, our analyses revealed that increasing severities (over-DIC, high PELOD-2 score, high PRISM-III score, nonsurvival) were associated with more hypocoagulable tendency in each EXTEM parameter (prolonged CT, CFT, but lower $\alpha$, MCF, TPI) compared with the less severe groups (nonovert DIC, low PELOD-2 score, low PRISM-III score, survival) (Supplementary Table). To the best of our knowledge, this is the 
TABLE 3 | Cutoff point of conventional parameters in the discrimination of hypocoagulable phase (based on rotational thromboelastometry).

\begin{tabular}{|c|c|c|c|c|c|c|}
\hline $\begin{array}{l}\text { Parameters } \\
\text { (Mean, IQR) }\end{array}$ & $\begin{array}{l}\text { Normo/hypercoagulable } \\
\qquad(n=32)\end{array}$ & $\begin{array}{l}\text { Hypocoagulable } \\
\qquad(n=23)\end{array}$ & $\begin{array}{c}\text { AUC } \\
(95 \% \mathrm{Cl})\end{array}$ & $\begin{array}{l}\text { Cutoff } \\
\text { (Se-Sp) }\end{array}$ & $p$-value & $\begin{array}{c}\text { OR } \\
(95 \% \mathrm{Cl})\end{array}$ \\
\hline PT (s) & 14.7 (12.9-19.6) & 20 (16.2-26.3) & $0.747(0.615-0.878)$ & 16.1 (82.6-68.7) & 0.002 & $10.5(2.8-38.8)$ \\
\hline INR & $1.2(1-1.5)$ & $1.6(1.4-2.4)$ & $0.754(0.624-0.884)$ & $1.4(78.3-68.7)$ & 0.001 & $6.9(2-23.5)$ \\
\hline Fibrinogen (g/L) & $3.9(3.6-5.3)$ & $2.6(1.5-4.2)$ & $0.728(0.582-0.874)$ & $3.3(69.6-81.2)$ & 0.004 & $9.9(2.8-34.8)$ \\
\hline D-dimer (ng/mL) & $2,371(1,617-3,801)$ & $6,935(2,343-17,172)$ & $0.728(0.588-0.867)$ & $3,863(65.2-78.1)$ & 0.004 & 6.7 (2-22.2) \\
\hline
\end{tabular}

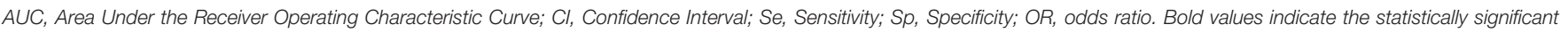
(p-value < 0.05).

IQR, Interquartile range; PLT, Platelet; PT, Prothrombin Time; INR, International Normalized Ratio; APTT, Activated Partial Thromboplastin Time.

first study comparing ROTEM parameters between the severity subgroups of the existing scoring system in pediatric patients. In previous septic studies in adults based on TEG/ROTEM, a higher sequential organ failure assessment (SOFA) score indicated a more hypocoagulable state than a lower SOFA score $(9,16,33)$.

Theoretically, sepsis-associated coagulopathy may lead to a variable clinical picture, ranging from hypercoagulopathy with microthrombosis to an increase in bleeding complications because of the secondary consumption of coagulation factors and platelets $(1,3,4)$. Therefore, identifying the coagulation profile is pivotal in timing anticoagulant therapy to both preserve the defense function of the hemostatic system and to avoid the harmful effects of overwhelming activation of coagulation. In fact, conventional coagulation tests are simply to be routinely utilized but are incapable of distinguishing the stages of hypercoagulability $(15,16)$. Table 2 shows no difference in most standard parameters (PT, INR, APTT, fibrinogen, D-dimer) between the normocoagulable and the hypercoagulable states. Although prolonged PT, INR, and APTT may occur, the profile on EXTEM would remain normocoagulable (Table 2). Prolonged PT, INR, and APTT, but high D-dimer, can lead to the misinterpretation of hyper- or hypocoagulability. In 2019, Saini et al. reported that hypercoagulability on TEG was more common than hypocoagulability; in contrast, hypocoagulability was more frequently observed on standard assays in children with severe sepsis (15). This can be explained as they rely on serum portions of the clotting cascade in isolation, and the PT and APTT only reflect clot formation partly. Therefore, a decrease in PT (\%) and prolongation of APTT are poor predictors of hemorrhage, which may overestimate the need for transfusion therapy $(34,35)$. Moreover, although patients had normal platelet count and fibrinogen level, the EXTEM may display a hypocoagulable profile (Table 2). This may be explained by the impaired platelet aggregation and fibrinogen function, which frequently manifest in sepsis $(11,12,36,37)$. Thus far, the contribution of both quantitative and functional platelets to clot strength was involved in ROTEM but eliminated in the standard assay. Interestingly, Table 3 shows that fibrinogen $<3.3 \mathrm{~g} / \mathrm{L}(\mathrm{AUC}=0.728, \mathrm{OR}=9.9$, $p=0.004)$ and $\mathrm{D}$-dimer $>3,863 \mathrm{ng} / \mathrm{mL}(\mathrm{AUC}=0.728, \mathrm{OR}=$ $6.7, p=0.004$ ) were the cutoff points corresponding with greater hazard of hypocoagulability on EXTEM. However, fibrinogen $<1(\mathrm{~g} / \mathrm{L})$ and D-dimer $>4,000(\mathrm{ng} / \mathrm{mL})$ are the thresholds according to the ISTH criteria for 2 and 3 points, respectively
(4). We strengthened the aforementioned hypothesis that using the ITSH criteria may delay the diagnosis of early phase or a hypercoagulable stage for the indication of anticoagulant therapy. Several studies in adult patients reported that the existing DIC scoring systems (such as ISTH, JAAM - Japanese Association for Acute Medicine) are valuable for fatal prognosis but late for timing diagnosis of "early asymptomatic DIC," "pre-DIC", "non-overt DIC," and "compensated DIC" (2, 38-40). Currently, the concept of SIC score combining platelet count, PT ratio, and total SOFA was recognized as the new tool for early coagulopathy detection, although with low specificity (39-41). This implies that DIC is initially characterized by widespread microvascular thrombosis induced in impaired organs; therefore, SOFA score describing both the number and the severity of organ dysfunction may allow early identification of the hypercoagulable stage for timely anticoagulant therapy. Nevertheless, further studies are required to investigate this issue on pediatric sepsis.

Our study has some limitations. A small number of participants were included from a single-center, and there was no existing standard definition of hypo- and hypercoagulability for ROTEM (17). Moreover, this study collected the coagulation parameters at a single time and did not evaluate their variations during the subsequent days. However, this may be beneficial as we can eliminate the effects of blood transfusion or anticoagulant therapy during treatment on hemostatic parameters. Nonetheless, further well-designed investigations should be undertaken to improve upon the findings of our study.

\section{CONCLUSIONS}

Hypocoagulable profile in patients with pediatric septic shock was associated with more severe conditions of the disease. Conventional tests may fail to detect hypercoagulability-the early stage of SIC, which is crucial in determining anticoagulation timing.

\section{DATA AVAILABILITY STATEMENT}

The original contributions presented in the study are included in the article/Supplementary Material, further inquiries can be directed to the corresponding author/s. 


\section{ETHICS STATEMENT}

The studies involving human participants were reviewed and approved by Institutional Review Board of Vietnam National Children's Hospital (approval no. 197-BVNTW-VNCSKTE). Written informed consent to participate in this study was provided by the participants' legal guardian/next of kin.

\section{AUTHOR CONTRIBUTIONS}

TT, NH, TX, TM, and TD contributed to the conception and design of the study. TT, LN, and $\mathrm{NH}$ collected the clinical information. $\mathrm{NH}$ wrote the first draft of the manuscript. TT, $\mathrm{NH}, \mathrm{TX}$, and TM wrote sections of the manuscript. $\mathrm{NH}$ and LN enrolled the patients, performed the coagulation test, and reviewed the manuscript. All authors have revised, read, and approved the final version of the manuscript.

\section{REFERENCES}

1. Simmons J, Pittet JF. The coagulopathy of acute sepsis. Curr Opin Anaesthesiol. (2015) 28:227-36. doi: 10.1097/ACO.0000000000000163

2. Scarlatescu E, Tomescu D, Arama SS. Anticoagulant therapy in sepsis. The importance of timing. J Crit Care Med. (2017) 3:63-9. doi: 10.1515/jccm-2017-0011

3. Levi M. The coagulant response in sepsis. Clin Chest Med. (2008) 29:62742. doi: $10.1016 /$ j.ccm.2008.06.006

4. Taylor FB, Toh CH, Hoots WK, Wada H, Levi M, Scientific Subcommittee on Disseminated Intravascular Coagulation (DIC) of the International Society on Thrombosis and Haemostasis (ISTH). Towards definition, clinical and laboratory criteria, and a scoring system for disseminated intravascular coagulation. Thromb Haemost. (2001) 86:1327-30. doi: 10.1055/s-0037-1616068

5. Di Nisio M, Baudo F, Cosmi B, D’Angelo A, De Gasperi A, Malato A, et al. Diagnosis and treatment of disseminated intravascular coagulation: guidelines of the Italian Society for Haemostasis and Thrombosis (SISET). Thromb Res. (2012) 129:e177-84. doi: 10.1016/j.thromres.2011.08.028

6. Davies GR, Lawrence M, Pillai S, Mills GM, Aubrey R, Thomas D, et al. The effect of sepsis and septic shock on the viscoelastic properties of clot quality and mass using rotational thromboelastometry: a prospective observational study. J Crit Care. (2018) 44:7-11. doi: 10.1016/j.jcrc.2017.09.183

7. Schmitt FCF, Manolov V, Morgenstern J, Fleming T, Heitmeier S, Uhle F, et al. Acute fibrinolysis shutdown occurs early in septic shock and is associated with increased morbidity and mortality: results of an observational pilot study. Ann Intensive Care. (2019) 9:19. doi: 10.1186/s13613-019-0499-6

8. Massion PB, Peters P, Ledoux D, Zimermann V, Canivet J-L, Massion PP, et al. Persistent hypocoagulability in patients with septic shock predicts greater hospital mortality: impact of impaired thrombin generation. Intensive Care Med. (2012) 38:1326-35. doi: 10.1007/s00134-012-2620-2

9. Ostrowski SR, Windeløv NA, Ibsen M, Haase N, Perner A, Johansson PI. Consecutive thrombelastography clot strength profiles in patients with severe sepsis and their association with 28-day mortality: a prospective study. J Crit Care. (2013) 28:317.e1-11. doi: 10.1016/j.jcrc.2012.09.003

10. Boscolo A, Spiezia L, Campello E, Bertini D, Lucchetta V, Piasentini E, et al. Whole-blood hypocoagulable profile correlates with a greater risk of death within 28 days in patients with severe sepsis. Korean J Anesthesiol. (2020) 73:224-31. doi: 10.4097/kja.19396

11. Boscolo A, Spiezia L, De Cassai A, Pasin L, Pesenti E, Zatta M, et al. Are thromboelastometric and thromboelastographic parameters associated with mortality in septic patients? A systematic review and meta-analysis. J Crit Care. (2021) 61:5-13. doi: 10.1016/j.jcrc.2020. 09.034

\section{FUNDING}

This work was supported, in part, by Grant-in-Aid from the Vietnam National University - Hanoi, Vietnam (QGSP.21.01 to TT).

\section{ACKNOWLEDGMENTS}

We would like to thank the Vietnam National University Hanoi (VNU - Hanoi) and the PICU staff at Vietnam National Children's Hospital.

\section{SUPPLEMENTARY MATERIAL}

The Supplementary Material for this article can be found online at: https://www.frontiersin.org/articles/10.3389/fped. 2021.676565/full\#supplementary-material

12. Zhou W, Zhou W, Bai J, Ma S, Liu Q, Ma X. TEG in the monitoring of coagulation changes in patients with sepsis and the clinical significance. Exp Ther Med. (2019) 17:3373-82. doi: 10.3892/etm.2019.7342

13. Wada H, Matsumoto $T$, Yamashita $Y$. Diagnosis and treatment of disseminated intravascular coagulation (DIC) according to four DIC guidelines. J Intensive Care. (2014) 2:15. doi: 10.1186/2052-0492-2-15

14. Halset JH, Hanssen SW, Espinosa A, Klepstad P. Tromboelastography: variability and relation to conventional coagulation test in nonbleeding intensive care unit patients. BMC Anesthesiol. (2015) 15:28. doi: 10.1186/s12871-015-0011-2

15. Saini A, Spinella PC, Ignell SP, Lin JC. Thromboelastography variables, immune markers, and endothelial factors associated with shock and NPMODS in children with severe sepsis. Front Pediatr. (2019) 7:422. doi: 10.3389/fped.2019.00422

16. Azim A, Muzaffar SN. Thromboelastography and thromboelastometry in patients with sepsis - a mini-review. J Anest Intern Care Med. (2017) 3:555603. doi: 10.19080/JAICM.2017.03.555603

17. Müller MC, Meijers JCM, Vroom MB, Juffermans NP. Utility of thromboelastography and/or thromboelastometry in adults with sepsis: a systematic review. Crit Care. (2010) 18:R30. doi: 10.1186/cc13721

18. Goldstein B, Giroir B, Randolph A, International Consensus Conference on Pediatric Sepsis. International pediatric sepsis consensus conference: definitions for sepsis and organ dysfunction in pediatrics. Pediatr Crit Care Med. (2005) 6:2-8. doi: 10.1097/01.PCC.0000149131.72248.E6

19. Tatman A, Warren A, Williams A, Powell JE, Whitehouse W. Development of a modified paediatric coma scale in intensive care clinical practice. Arch Dis Child. (1997) 77:519-21. doi: 10.1136/adc.77.6.519

20. Pollack MM, Patel KM, Ruttimann UE. PRISM III: an updated pediatric risk of mortality score. Crit Care Med. (1996) 24:74352. doi: 10.1097/00003246-199605000-00004

21. Leteurtre S, Duhamel A, Salleron J, Grandbastien B, Lacroix J, Leclerc F, et al. PELOD-2: an update of the PEdiatric logistic organ dysfunction score. Crit Care Med. (2013) 41:1761-73. doi: 10.1097/CCM.0b013e31828a2bbd

22. Brenner T, Schmidt K, Delang M, Mehrabi A, Bruckner T, Lichtenstern C, et al. Viscoelastic and aggregometric point-of-care testing in patients with septic shock - cross-links between inflammation and haemostasis. Acta Anaesthesiol Scand. (2012) 56:1277-90. doi: 10.1111/j.1399-6576.2012.02750.x

23. Andersen MG, Hvas CL, Tønnesen E, Hvas A-M. Thromboelastometry as a supplementary tool for evaluation of hemostasis in severe sepsis and septic shock. Acta Anaesthesiol Scand. (2014) 58:525-33. doi: 10.1111/aas.12290

24. Boyd CJ, Claus MA, Raisis AL, Hosgood G, Sharp CR, Smart L. Hypocoagulability and platelet dysfunction are exacerbated by synthetic colloids in a canine hemorrhagic shock model. Front Vet Sci. (2018) 5:279. doi: $10.3389 /$ fvets.2018.00279 
25. Lang T, Bauters A, Braun SL, Pötzsch B, von Pape K-W, Kolde $\mathrm{H}-\mathrm{J}$, et al. Multi-centre investigation on reference ranges for ROTEM thromboelastometry. Blood Coagul Fibrinolysis. (2005) 16:301-10. doi: 10.1097/01.mbc.0000169225.31173.19

26. Mandrekar JN. Receiver operating characteristic curve in diagnostic test assessment. J Thorac Oncol. (2010) 5:13156. doi: 10.1097/JTO.0b013e3181ec173d

27. Muzaffar SN, Baronia AK, Azim A, Verma A, Gurjar M, Poddar B, et al. Thromboelastography for evaluation of coagulopathy in nonbleeding patients with sepsis at intensive care unit admission. Indian J Crit Care Med. (2017) 21:268-73. doi: 10.4103/ijccm.IJCCM_72_17

28. Donzé JD, Ridker PM, Finlayson SRG, Bates DW. Impact of sepsis on risk of postoperative arterial and venous thromboses: large prospective cohort study. BMJ. (2014) 349:g5334. doi: 10.1136/bmj.g5334

29. Sivula M, Pettilä V, Niemi TT, Varpula $M$, Kuitunen AH. Thromboelastometry in patients with severe sepsis and disseminated intravascular coagulation. Blood Coagul Fibrinolysis. (2009) 20:419-26. doi: 10.1097/MBC.0b013e32832a76e1

30. Scarlatescu E, White NJ, Tomescu DR. Standard and derived rotational thromboelastometry parameters for prediction of disseminated intravascular coagulation in septic patients. Blood Coagul Fibrinolysis. (2020) 31:31723. doi: 10.1097/MBC.0000000000000919

31. Johansson PI, Stensballe J, Vindeløv N, Perner A, Espersen K. Hypocoagulability, as evaluated by thrombelastography, at admission to the ICU is associated with increased 30-day mortality. Blood Coagul Fibrinolysis. (2010) 21:168-74. doi: 10.1097/MBC.0b013e328 3367882

32. Sokou R, Giallouros G, Konstantinidi A, Pantavou K, Nikolopoulos G, Bonovas S, et al. Thromboelastometry for diagnosis of neonatal sepsisassociated coagulopathy: an observational study. Eur J Pediatr. (2018) 177:355-62. doi: 10.1007/s00431-017-3072-z

33. Daudel F, Kessler U, Folly H, Lienert JS, Takala J, Jakob SM. Thromboelastometry for the assessment of coagulation abnormalities in early and established adult sepsis: a prospective cohort study. Crit Care. (2009) 13:R42. doi: 10.1186/cc7765

34. Gajic O, Dzik WH, Toy P. Fresh frozen plasma and platelet transfusion for nonbleeding patients in the intensive care unit: benefit or harm? Crit Care Med. (2006) 34:S170-3. doi: 10.1097/01.CCM.0000214288.88308.26
35. Chowdary P, Saayman AG, Paulus U, Findlay GP, Collins PW. Efficacy of standard dose and $30 \mathrm{ml} / \mathrm{kg}$ fresh frozen plasma in correcting laboratory parameters of haemostasis in critically ill patients. Br J Haematol. (2004) 125:69-73. doi: 10.1111/j.1365-2141.2004.04868.x

36. Yaguchi A, Lobo FLM, Vincent J-L, Pradier O. Platelet function in sepsis. J Thromb Haemost. (2004) 2:2096-102. doi: 10.1111/j.1538-7836.2004.01009.x

37. Alt E, Amann-Vesti BR, Madl C, Funk G, Koppensteiner R. Platelet aggregation and blood rheology in severe sepsis/septic shock: relation to the Sepsis-related Organ Failure Assessment (SOFA) score. Clin Hemorheol Microcirc. (2004) 30:107-15.

38. Yamakawa K, Umemura Y, Murao S, Hayakawa M, Fujimi S. Optimal timing and early intervention with anticoagulant therapy for sepsis-induced disseminated intravascular coagulation. Clin Appl Thromb Hemost. (2019) 25:1076029619835055. doi: 10.1177/1076029619835055

39. Iba T, Levy JH, Raj A, Warkentin TE. Advance in the management of sepsisinduced coagulopathy and disseminated intravascular coagulation. JClin Med. (2019) 8:728. doi: $10.3390 / \mathrm{jcm} 8050728$

40. Helms J, Severac F, Merdji H, Clere-Jehl R, François B, Mercier E, et al. Performances of disseminated intravascular coagulation scoring systems in septic shock patients. Ann Intensive Care. (2020) 10:92. doi: 10.1186/s13613-020-00704-5

41. Ding R, Wang Z, Lin Y, Liu B, Zhang Z, Ma X. Comparison of a new criteria for sepsis-induced coagulopathy and International Society on Thrombosis and Haemostasis disseminated intravascular coagulation score in critically ill patients with sepsis 3.0: a retrospective study. Blood Coagul Fibrinolysis. (2018) 29:551-8. doi: 10.1097/MBC.000000000000075

Conflict of Interest: The authors declare that the research was conducted in the absence of any commercial or financial relationships that could be construed as a potential conflict of interest.

Copyright (C) 2021 Tuan, Ha, Xoay, My, Nghiem and Dien. This is an open-access article distributed under the terms of the Creative Commons Attribution License (CC $B Y)$. The use, distribution or reproduction in other forums is permitted, provided the original author(s) and the copyright owner(s) are credited and that the original publication in this journal is cited, in accordance with accepted academic practice. No use, distribution or reproduction is permitted which does not comply with these terms. 